\section{Lung macrophages: old hands required rather than new blood?}

\author{
Karl J Staples ${ }^{1,2}$
}

Macrophages are key sentinel cells of the lung, clearing inhaled particulates and micro-organisms from the airway. These cells perform this function without provoking inflammatory responses that could lead to pneumonias. Previous work suggested that the lung macrophage population was maintained by the recruitment of blood monocytes. ${ }^{1}$ However, this observation was made in transplanted lungs where macrophages had been depleted, either by hypoxia or chemotherapy. More recent work in murine models using cell fate mapping demonstrated that lung macrophages are generated early in embryonic development initially in the foetal yolk sac and slightly later in the foetal liver. ${ }^{2}$ Macrophages that arise from these sources then seed the lung, where they reside as a self-renewing population with little further recruitment from blood monocytes.

The relevance and translation of these murine findings to the ontogeny of human lung macrophages has remained unclear. The study by Eguíluz-Gracia et $a l^{3}$ has provided some intriguing evidence regarding the stability and longevity of the lung macrophage population. This study prospectively followed 10 gender mismatched lung transplant patients for 2 years. Transbronchial biopsies were collected at regular intervals throughout the follow-up period. The macrophage populations in these biopsies were analysed for known macrophage markers, indicators of proliferation and, importantly, in situ hybridisation for the $\mathrm{X}$ and $\mathrm{Y}$ chromosomes to differentiate between recipient and donor cells. Comparisons to normal lung tissue obtained from cancer resections, suggest that there is no significant difference in the total number of macrophages in

\footnotetext{
${ }^{1}$ Department of Clinical and Experimental Sciences, University of Southampton Faculty of Medicine, Sir Henry Wellcome Laboratories, Southampton General Hospital, Southampton, UK; ${ }^{2}$ Wessex Investigational Sciences Hub, University of Southampton Faculty of Medicine, Southampton General Hospital,

Southampton, UK
}

Correspondence to Dr Karl J Staples, Department of Clinical \& Experimental Sciences, University of Southampton Faculty of Medicine, Sir Henry Wellcome Laboratories, Mailpoint 810, Southampton General Hospital, Tremona Road, Southampton S016 6YD, UK; k.staples@southampton.ac.uk transplanted lung tissue across the 2 years. Moreover, while there was a large amount of inter-individual variation the abundance of donor-derived macrophages was largely unchanged over the follow-up period. At the same time, there was a rapid infiltration of recipient-derived macrophages that, once established, also remained stable.

To investigate whether these infiltrating cells were recruited blood monocytes, Eguíluz-Gracia et $a l^{3}$ analysed the coexpression of the macrophage markers CD68 and DC-SIGN (CD209) and the monocyte marker calprotectin. Calprotectin was only detected on CD68+DC-SIGN+ macrophages at 2 weeks post-transplantation but not at 2 years post-transplantation. Taken together these results provided circumstantial evidence that blood monocytes were being recruited initially to the transplanted lung before maturing into macrophages as they became resident in the tissue. The investigators then went a step further to prove that recruited monocytes did indeed differentiate into lung macrophages by injecting human CD34+ cord blood cells into humanised mice that had been sublethally irradiated. The lungs of these animals were then harvested after 10 weeks and macrophages analysed for the presence of human antigens. In line with the human findings, the macrophages in these mouse lungs expressed the human CD45, CD68 and DC-SIGN antigens.

While these experiments demonstrated that monocytes were recruited to the murine lung, the loss of calprotectin over the 2 years suggested that this recruitment was not a common occurrence in the human lung. The authors therefore questioned whether recruited monocyte-derived lung macrophages could go on to proliferate to maintain this population. Eguíluz-Gracia and colleagues observed expression of the Ki67 protein marker of cellular proliferation in a median of $2 \%$ of the CD68+ macrophage population and that this proportion of Ki67 expressing cells remained stable over the 2 years. Interestingly, both donor and recipient macrophages were observed to express $\mathrm{Ki67}$, although accurate quantitation of the number of proliferating donor cells was precluded due to low numbers of donor- derived Ki67+ macrophages. These results suggest that donor cells can persist in the lung for at least 2 years and some of this longevity may be due to proliferation of these macrophages. Furthermore, blood monocytes can replenish the lung if alveolar macrophages are depleted in some way, but once the lung is refilled macrophage numbers may once again be maintained by local proliferation rather than further recruitment from the monocyte pool.

The biggest limitation of this study is that it is impossible to definitively identify whether the persistence of the macrophage population is due to cellular longevity or self-renewal, that is, the $2 \%$ of cells identified as proliferating give rise to the other $98 \%$ of macrophages. As with any study using human patients rather than murine models, there is substantial inter-individual variation in the sex, underlying disease and chemotherapy of the patients. However, none of these factors appeared to be related to the variation in cell densities. A further possibility is that the lack of significant changes in donor macrophage numbers could be confounded by the small numbers of subjects included. However, given the logistical issues in running such a study, not least persuading patients to return to hospital for transbronchial biopsies five times posttransplant, the authors should be commended on the quality of the data obtained. One further possible contribution to the inter-individual variation, also acknowledged by the authors, is that during counting only $50 \%$ of the cells could be called as having two sex chromosomes and thus being identified as a donor or recipient macrophage. However, the authors present data from biopsies sampled at the early time points demonstrating that the proportions of chromosome determined and undetermined cells were close to $50 \%$ in all samples examined, so this is unlikely to have skewed the cell counts.

The authors discuss the implications of this work in terms of its applicability for the use of donor monocyte-derived macrophages to replace defective macrophages in patients with alveolar proteinosis. $^{4}$ However, this work also has implications for lung macrophage biology outside of the transplantation field, and COPD in particular. Macrophage numbers are increased in lung tissue taken from patients with COPD and the number of airways containing macrophages increases in line with disease severity. ${ }^{5}$ While numbers are increased, there are reports of macrophages being less mature in COPD in terms of both size $^{6}$ and 
surface marker expression. ${ }^{7}$ Taken together with the increased expression of the chemokine monocyte chemoattractant protein-1 (CCL2) described in the COPD airway, ${ }^{8}$ these observations suggest that monocytes may be recruited to the COPD airway. The question remains what do these newly recruited cells do in the COPD lung and what is recruiting them?

The development of mature lung macrophages is controlled by a number of factors including granulocyte/macrophage colony-stimulating factor as well as interactions with the lung microbiome acquired early in life. The microbiome has been shown to play a role in susceptibility to inflammatory diseases of the gut and recent work implicates dysbiosis of the lung microbiome in a number of conditions including COPD (reviewed in ref. 9). Resident macrophages may be largely tolerant of the normal lung microbiota through recognition of bacterialderived short chain fatty acids as in the gut. ${ }^{10}$ It is possible that newly recruited macrophages have been recruited and 'trained' by the milieu of the dysbiotic airway to either not recognise potentially pathogenic microorganisms in this environment or respond to these bacteria inappropriately which may explain the dysregulation of these cells in COPD. For example, a recent study of the transcriptome of macrophages from the COPD lung revealed a diminution of inflammatory responses. ${ }^{11}$ Supporting this observation of reduced macrophage responses, we have demonstrated that COPD macrophages do not express the regulatory ligand PD-L1 in response to viral infection, ${ }^{12}$ which may indirectly lead to increased inflammation of the COPD airway.
The scenario described above may not be limited to COPD as both macrophages $^{13}$ and microbiome changes ${ }^{9}$ are also evident in many lung pathologies. However, more evidence will be required to support these speculations and, based on the work by Eguíluz-Gracia et $a l{ }^{3}$ we now have a firm rationale for generating such data. The recent description of markers for macrophage subsets in the human lung may enable us to sort the resident from the recruited cells. ${ }^{14}$ When combined with our ability to analyse macrophage gene expression and the lung microbiome to a greater level than ever before, we are on the brink of beginning to understand these complex interactions between macrophage, disease and microbiome and what they may mean for disease pathogenesis and progression.

Competing interests None declared.

Provenance and peer review Commissioned; externally peer reviewed.

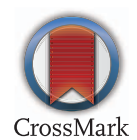

To cite Staples KJ. Thorax 2016;71:973-974.

Published Online First 16 August 2016

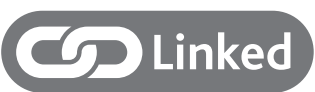

- http://dx.doi.org/10.1136/thoraxjnl-2016-208292

Thorax 2016:71:973-974.

doi:10.1136/thoraxjnl-2016-208992

\section{REFERENCES}

1 Thomas ED, Ramberg RE, Sale GE, et al. Direct evidence for a bone marrow origin of the alveolar macrophage in man. Science 1976;192:1016-18.
2 Yona S, Kim KW, Wolf $\mathrm{Y}$, et al. Fate mapping reveals origins and dynamics of monocytes and tissue macrophages under homeostasis. Immunity 2013:38:79-91.

3 Eguíluz-Gracia I, Schultz HH, Sikkeland LI, et al. Long-term persistence of human donor alveolar macrophages in lung transplant recipients. Thorax 2016;71:1006-11.

4 Doerschuk CM. Pulmonary alveolar proteinosis and macrophage transplantation. N Engl J Med 2015;372: 1762-4.

5 Hogg JC, Chu F, Utokaparch S, et al. The nature of small-airway obstruction in chronic obstructive pulmonary disease. N Engl J Med 2004;350: 2645-53.

6 Frankenberger $M$, Menzel M, Betz $R$, et al. Characterization of a population of small macrophages in induced sputum of patients with chronic obstructive pulmonary disease and healthy volunteers. Clin Exp Immunol 2004;138:507-16.

7 Chana KK, Fenwick PS, Nicholson AG, et al. Identification of a distinct glucocorticosteroid-insensitive pulmonary macrophage phenotype in patients with chronic obstructive pulmonary disease. J Allergy Clin Immunol 2014;133:207-16.e1-11.

8 Traves SL, Culpitt SV, Russell RE, et al. Increased levels of the chemokines GROalpha and MCP-1 in sputum samples from patients with COPD. Thorax 2002;57:590-5.

9 O'Dwyer DN, Dickson RP, Moore BB. The lung microbiome, immunity, and the pathogenesis of chronic lung disease. J Immunol 2016;196:4839-47.

10 Chang PV, Hao L, Offermanns S, et al. The microbial metabolite butyrate regulates intestinal macrophage function via histone deacetylase inhibition. Proc Natl Acad Sci USA 2014;111:2247-52.

11 Xue J, Schmidt SV, Sander J, et al. Transcriptome-based network analysis reveals a spectrum model of human macrophage activation. Immunity 2014;40:274-88.

12 McKendry RT, Spalluto CM, Burke $\mathrm{H}$, et al. Dysregulation of antiviral function of CD8(+) T cells in the chronic obstructive pulmonary disease lung. Role of the PD-1-PD-L1 axis. Am J Respir Crit Care Med 2016;193:642-51.

13 Byrne AJ, Mathie SA, Gregory LG, et al. Pulmonary macrophages: key players in the innate defence of the airways. Thorax 2015;70:1189-96.

14 Desch AN, Gibbings SL, Goyal R, et al. Flow cytometric analysis of mononuclear phagocytes in nondiseased human lung and lung-draining lymph nodes. Am J Respir Crit Care Med 2016;193:614-26. 